\title{
Critical parameters for non-hermitian Hamiltonians
}

\author{
Francisco M Fernández and Javier Garcia \\ INIFTA (UNLP, CCT La Plata-CONICET), División Química Teórica, Blvd. 113 \\ S/N, Sucursal 4, Casilla de Correo 16, 1900 La Plata, Argentina \\ E-mail: fernande@quimica.unlp.edu.ar
}




\begin{abstract}
We calculate accurate critical parameters for a class of non-hermitian Hamiltonians by means of the diagonalization method. We study three one-dimensional models and two perturbed rigid rotors with PT symmetry. One of the latter models illustrates the necessity of a more general condition for the appearance of real eigenvalues that we also discuss here.
\end{abstract}

\title{
1. Introduction
}

There has recently been interest in PT-symmetric Hamiltonians that exhibit real eigenvalues for a range of values of a potential parameter. Some of them are anharmonic oscillators [1-9] as well as models with Dirichlet [10 12 periodic and anti-periodic boundary conditions [13, 14].

Among the methods used for the study of such models we mention the WKB approximation [3, 4], the eigenvalue moment method [5, 7], the multiscale reference function analysis [6], the diagonalization method (DM) [8] and the orthogonal polynomial projection quantization (OPPQ) (an improved Hill-determinant method) [9].

For some particular values of the potential parameter the spectrum of those PTsymmetric Hamiltonians exhibits critical points where two real eigenvalues coalesce and emerge as complex conjugate eigenvalues. Such critical points are also known as exceptional points $[15-18]$.

The purpose of this paper is the analysis of the critical points for a variety of simple models. The calculation is based on a well known simple and quite efficient application of the DM [15]. In section 2 we propose a somewhat more general condition for the existence of real eigenvalues (unbroken symmetry) [19,20] that is suitable for models with degenerate states. In section 3 we present three one-dimensional examples already discussed earlier by other authors. In section 4 we outline the procedure for the calculation of critical points based on the DM. In section 5 we apply perturbation theory to one of the models and discuss the convergence of the perturbation series for the eigenvalues by comparison with the accurate results produced by the DM. In section 6 
we discuss a PT-symmetric perturbed planar rigid rotor that was studied earlier as an example with E2 algebra [14]. In section [7 we discuss a non-hermitian perturbed three-dimensional rigid rotor that was not treated before as far as we know. This most interesting model illustrates the generalized condition for real eigenvalues mentioned above. Finally, in section 8 we summarize the main results and draw conclusions.

\section{PT Symmetry}

It is well known that a wide class of non-hermitian Hamiltonians with unbroken PT symmetry exhibit real spectra [19,20]. In general, they are invariant under an antilinear or antiunitary transformation of the form $\hat{A}^{-1} \hat{H} \hat{A}=\hat{H}$. The antiunitary operator $\hat{A}$ satisfies [21]

$$
\begin{aligned}
\hat{A}(|f\rangle+|g\rangle) & =\hat{A}|f\rangle+\hat{A}|g\rangle \\
\hat{A} c|f\rangle & =c^{*} \hat{A}|f\rangle,
\end{aligned}
$$

for any pair of vectors $|f\rangle$ and $|g\rangle$ and arbitrary complex number $c$, where the asterisk denotes complex conjugation. This definition is equivalent to

$$
\langle\hat{A} f \mid \hat{A} g\rangle=\langle f \mid g\rangle^{*}
$$

It follows from the antiunitary invariance mentioned above that $[\hat{H}, \hat{A}]=0$. Therefore, if $|\psi\rangle$ is an eigenvector of $\hat{H}$ with eigenvalue $E$

$$
\hat{H}|\psi\rangle=E|\psi\rangle
$$

we have

$$
[\hat{H}, \hat{A}]|\psi\rangle=\hat{H} \hat{A}|\psi\rangle-\hat{A} \hat{H}|\psi\rangle=\hat{H} \hat{A}|\psi\rangle-E^{*} \hat{A}|\psi\rangle=0
$$

This equation merely tell us that if $|\psi\rangle$ is eigenvector of $\hat{H}$ with eigenvalue $E$ then $\hat{A} \hat{H}|\psi\rangle$ is eigenvector with eigenvalue $E^{*}$. Consequently, $E$ is real if

$$
\hat{H} \hat{A}|\psi\rangle=E \hat{A}|\psi\rangle,
$$

that contains the condition of unbroken symmetry required by Bender et al [19, 20]

$$
\hat{A}|\psi\rangle=\lambda|\psi\rangle
$$


as a particular case. Note that equation (5) applies to the case in which $\hat{A}|\psi\rangle$ is a linear combination of degenerate eigenvectors of $\hat{H}$ with eigenvalue $E$.

If $\hat{K}$ is an antilinear operator such that $\hat{K}^{2}=\hat{1}$ (for example, the complex conjugation operator) then it follows from (2) that $\hat{A} \hat{K}=\hat{U}$ is unitary $\left(\hat{U}^{\dagger}=\hat{U}^{-1}\right)$. In other words, any antilinear operator $\hat{A}$ can be written as a product of a unitary operator and the complex conjugation operation [21]. In most of the non-hermitian models studied $\hat{U}^{-1}=\hat{U}$ that results in $\hat{A}^{2}=\hat{1}$ (as in the case of the parity operator $\hat{U}=\hat{P}$ that gives rise to PT symmetry) [19,20].

\section{Some simple one-dimensional examples}

In this section we consider three examples of the Schrödinger equation

$$
\begin{aligned}
& \hat{H} \psi=E \psi \\
& \hat{H}=\hat{p}^{2}+\hat{V}(x),
\end{aligned}
$$

with eigenvalues $E_{0}<E_{1}<\ldots$

The first one $[3,5,6]$

$$
\hat{H}=\hat{p}^{2}+i \hat{x}^{3}+i a \hat{x}
$$

exhibits an infinite set of critical values $0>a_{0}>a_{1}>\ldots>a_{n}>\ldots$ of $a$ so that $E_{2 n}=E_{2 n+1}$ at $a=a_{n}$. Both eigenvalues are real when $a>a_{n}$ and become complex conjugate numbers when $a<a_{n}$. The eigenfunctions $\psi_{2 n}$ and $\psi_{2 n+1}$ are linearly dependent at the exceptional point $a=a_{n}$ [15 18].

The second example is [1,4, 7 ]

$$
\hat{H}=\hat{p}^{2}+\hat{x}^{4}+i a \hat{x}
$$

If $\hat{P}$ denotes the parity operator we have $\hat{P} \hat{H}(a) \hat{P}=\hat{H}(-a)$ so that $E(-a)=E(a)$. Because of this property of the eigenvalues the crossings $E_{2 n}=E_{2 n+1}$ take place at $\pm a_{n}$, where $0<a_{0}<a_{1}<\ldots<a_{n}<\ldots$ In this case the pair of coalescing eigenvalues become complex conjugate numbers when $|a|>a_{n}$ 
The third example is given by

$$
\hat{H}=\hat{p}^{2}+i a \hat{x}
$$

with the boundary conditions $\psi( \pm 1)=0$. In this case we also find that the crossings take place at $\pm a_{n}, a_{n}>0$ as in the preceding one. Because of physical reasons Rubinstein et al [10] considered only the half line $a>0$.

\section{Diagonalization method}

In order to solve the Schrödinger equation (7) we resort to a matrix representation of the Hamiltonian operator $H_{i j}=\langle i|\hat{H}| j\rangle$ in an appropriate orthonormal basis set $\{|j\rangle, j=0,1, \ldots\}$. We obtain the eigenvalues from the roots of the characteristic polynomial given by the secular determinant $D(E, a)=|\mathbf{H}-E \mathbf{I}|=0$, where $\mathbf{H}$ is an $N \times N$ matrix with elements $H_{i j}, i, j=0,1, \ldots, N-1$ and $\mathbf{I}$ is the $N \times N$ identity matrix. We look for those roots of the characteristic polynomial that converge as $N$ increases. The characteristic polynomial gives us either $E(a)$ or $a(E)$.

In all the examples discussed here the critical parameters are given by $a_{n}=a\left(e_{n}\right)$, where

$$
\left.\frac{d a}{d E}\right|_{E=e_{n}}=0
$$

and $E_{2 n}\left(a_{n}\right)=E_{2 n+1}\left(a_{n}\right)=e_{n}$. Therefore, we can obtain the critical parameters approximately from the set of polynomial equations $\{D(E, a)=0, \partial D(E, a) / \partial E=0\}$ [15]. We look for pairs of roots $\left(a_{n, N}, e_{n, N}\right)$ that converge as $N \rightarrow \infty$.

The eigenvectors of the harmonic oscillator $\hat{H}_{0}=\hat{p}^{2}+\hat{x}^{2}$ are a suitable basis set for the first two examples (8) and (9), and for the third one (10) we choose

$$
\phi_{n}(x)=\langle x \mid n\rangle=\sin \left(\frac{n \pi(x+1)}{2}\right), n=1,2, \ldots
$$

Before proceeding with the discussion of the examples we want to stress that the DM is a simple and most efficient approach for the accurate calculation of the eigenvalues and eigenfunctions of those PT-symmetric oscillators with eigenfunctions that vanish exponentially along the real $x$ axis. In order to illustrate this point we compare the DM 
with the recently developed OPPQ [9]. As an example we choose the PT-symmetric oscillator $\hat{H}=\hat{p}^{2}+i \hat{x}^{3}$ because Handy and Vrinceanu [9] showed OPPQ results of increasing order of accuracy for this model. Although both methods resort to the same Gaussian function and Hermite polynomials, Table 1 shows that the rate of convergence of the DM is noticeably greater. It is striking that the DM of order $N$ appears to be nearly as accurate as the OPPQ of order $N+20$.

Tables 2, 3 and 4 show the first critical parameters for the examples (8), (9) and (10) calculated with $N \leq 300, N \leq 300$ and $N=100$ basis functions, respectively. With those results we carried out nonlinear regressions of the form

$$
a_{n}=b+c e_{n}^{s}
$$

and obtained

$$
\begin{aligned}
& b=-0.324 \pm 0.015 \\
& c=-1.9288 \pm 0.0083 \\
& s=0.6751 \pm 0.0011,
\end{aligned}
$$

for (8),

$$
\begin{aligned}
& b=0.407 \pm 0.010 \\
& c=1.1540 \pm 0.0048 \\
& s=0.7555 \pm 0.0010,
\end{aligned}
$$

for (9) and

$$
\begin{aligned}
& b=-0.00028 \pm 0.00016 \\
& c=1.732092 \pm 9.5 \times 10^{-6} \\
& s=0.9999951 \pm 9.4 \times 10^{-7},
\end{aligned}
$$

for (10). The parameters in equation (15) are in good agreement with the WKB ones [4] which suggests that even the first critical parameters for that model exhibit the large- $e_{n}$ 
asymptotic behaviour given exactly by the WKB method. The nonlinear regression appears to be most accurate for the example (10) where it seems that $a_{n}=1.7321 e_{n}$. It seems that $e_{n}$ is always approximately between $E_{2 n-1}(a=0)$ and $E_{2 n}(a=0)$ and, therefore, increases asymptotically as $n^{2}$. Consequently, $a_{n}$ behaves approximately in the same way.

In the discussion below we sometimes find it convenient to write $g$ for $i a$ and consider $g$ complex. Figure 1] shows $E_{n}(g), n=1,2,3,4$ for the example (10) for $g$ real and purely imaginary. We will discuss this case with more detail in the next section.

\section{Perturbation theory}

Delabaere and Trinh [3] derived the exact Rayleigh-Schrödinger series asymptotic to the eigenvalues of the Hamiltonian (8) for large $a$. For the three examples discussed in section 3 it is also possible to obtain a perturbation series for small $a$ (see, for example, Fernández et al [2]). In all of them the Taylor series for $E_{n}$ about $a=0$ exhibits a finite nonzero radius of convergence (see, for example, page 111 in reference [23] and references therein). The three Hamiltonians are PT symmetric when $g$ is imaginary and (9) and (10) are Hermitian when $g$ is real. The perturbation series for the eigenvalues of either (9) or (10) reads

$$
E_{n}(g)=\sum_{j=0}^{\infty} E_{n, j} g^{2 j} .
$$

We can calculate the coefficients $E_{n, j}$ approximately for the former and exactly for the latter. By means of a variety of well known methods [23] we easily obtain

$$
\begin{aligned}
E_{n}= & \frac{1}{2} b_{n}+\frac{\left(2 b_{n}-15\right) g^{2}}{12 b_{n}^{2}}+\frac{\left(b_{n}^{2}-105 b_{n}+495\right) g^{4}}{18 b_{n}^{5}} \\
& +\frac{\left(2 b_{n}^{3}-825 b_{n}^{2}+23400 b_{n}-95625\right) g^{6}}{36 b_{n}^{8}}+\ldots,
\end{aligned}
$$

where $b_{n}=n^{2} \pi^{2} / 2$. The radius of convergence of the perturbation series for both $E_{2 n-1}$ and $E_{2 n}, n=1,2, \ldots$ cannot be greater than $a_{n}$ because the two eigenvalues coalesce at the exceptional branch points $g= \pm i a_{n}$. 
Figure 2 shows the first four eigenvalues of the problem (10) when $g=i a$ calculated by means of the DM and by perturbation theory of order 20 . We appreciate that there is a good agreement between both approaches for the first two eigenvalues for almost all the values of $-a_{1}<a<a_{1}$ except close to the crossings where perturbation theory is expected to fail. The situation appears to be quite similar for the fourth eigenvalue but the behaviour of the perturbation series for the third eigenvalue strongly suggests that its radius of convergence may be considerably smaller than $a_{2}$.

If $g= \pm i a_{n}$ were the singularities closest to the origin, one could obtain them from the perturbation coefficients $E_{n, j}$ as follows: [23]

$$
a_{n}=\lim _{k \rightarrow \infty}\left|\frac{(1 / 2-k) E_{2 n-1, k}}{(k+1) E_{2 n-1, k+1}}\right|^{1 / 2}=\lim _{k \rightarrow \infty}\left|\frac{(1 / 2-k) E_{2 n, k}}{(k+1) E_{2 n, k+1}}\right|^{1 / 2} .
$$

Table 5 shows that $a_{1}(k)=\left|\frac{(1 / 2-k) E_{1, k}}{(k+1) E_{1, k+1}}\right|^{1 / 2}$ already converges towards the result in Table 4 as $k$ increases, and we obtain identical results with the coefficient $E_{2, k}$ as expected. However, the sequences with $E_{n, k}$ do not converge when $n>2$ which suggests that there may be other branch points on the complex $g$-plane closest to the origin. For example, $E_{3}(g)$ exhibits branch points at $g_{c}= \pm 11.48088661+26.24188126 i$ and also at $g_{c}^{*}$ that are closer to the origin than $g_{2}=i a_{2}\left(\left|g_{c}\right|=28.64344759<a_{2}\right)$. As already mentioned above, equation (19) is only suitable for a branch point on the imaginary axis [23] and therefore does not converge in the latter case. The branch points at $g_{c}$ and $g_{c}^{*}$ account for the behaviour of the perturbation series for $E_{3}(g)$ in Fig. 2 discussed above.

\section{Non-hermitian perturbed planar rigid rotor}

In this section we consider a simple model with periodic boundary conditions that we prefer to treat separately from those in section 3 ,

Bender and Kalvecks [14] studied the eigenvalues of

$$
-\psi^{\prime \prime}(\theta)+g \cos (\theta) \psi(\theta)=E \psi(\theta)
$$

with periodic $\psi(\theta+2 \pi)=\psi(\theta)$ and anti-periodic $\psi(\theta+2 \pi)=-\psi(\theta)$ boundary 
conditions. This equation is a particular case of [14]

$$
\begin{aligned}
& \hat{H} \psi=E \psi, \\
& \hat{H}=\hat{J}^{2}+V(g, \theta), \hat{J}=-i \frac{d}{d \theta},
\end{aligned}
$$

when $V(g, \theta)=g \cos (\theta)$.

By means of the unitary operator $\hat{U}$ that produces the transformation $\hat{U}^{\dagger} \theta \hat{U}=$ $\theta+\pi, \hat{U}^{\dagger} \hat{J} \hat{U}=\hat{J}$ we can construct the antiunitary operator $\hat{A}=\hat{U} \hat{T}=\hat{T} \hat{U}$, where $\hat{T}$ is the time-inversion operator, as indicated in section 2. Since $A^{-1} \hat{H} \hat{A}=\hat{H}$ when $g=i a$ is purely imaginary we expect real eigenvalues for some real values of $a$.

Here we consider only periodic boundary conditions and transform equation (20) into the Mathieu equation [22] by means of the transformations $\theta=2 x, E^{B K}=E / 4$ and $g^{B K}=g / 2$, so that

$$
\varphi^{\prime \prime}(x)+[E-2 g \cos (2 x)] \varphi(x)=0,
$$

where $\varphi(x)=\psi(2 x)$. The even and odd solutions to this equation can be expanded in the Fourier series

$$
\begin{aligned}
& \varphi_{e}(x)=\sum_{m=0}^{\infty} A_{2 m} \cos (2 m x), \\
& \varphi_{o}(x)=\sum_{m=1}^{\infty} B_{2 m} \sin (2 m x),
\end{aligned}
$$

respectively, where the coefficients $A_{2 m}$ and $B_{2 m}$ can be calculated by means of simple three-term recurrence relations [22]. We can efficiently calculate accurate eigenvalues from either the secular determinant, as discussed in section 4, or the truncation conditions $A_{2 N}=0$ and $B_{2 N}=0$ for sufficiently large values of $N$. We denote $E_{e, n}$ $n=0,1, \ldots$ and $E_{o, n}, n=1,2, \ldots$ the eigenvalues of the even and odd solutions, respectively. Obviously, $E_{e, n}=E_{o, n}=4 n^{2}, n=1,2, \ldots$, when $g=0$.

The results of Bender and Kalveks [14] suggest that pairs of eigenvalues $\left(E_{e, 2 n}, E_{e, 2 n+1}\right)$ and $\left(E_{o, 2 n+1}, E_{o, 2 n}\right), n=0,1, \ldots$ coalesce at $\pm a_{e, n}$ and $\pm a_{o, n}$, respectively, when $g=i a$. Tables 6 and 7 show the critical parameters for the even and odd solutions, respectively, to the Mathieu equation (22). They approximately follow a straight line of 
the form $a_{n}=0.582 e_{n}+3.66$. Once again we appreciate that both $e_{n}$ and $a_{n}$ increase asymptotically as $n^{2}$.

\section{Non-hermitian perturbed three-dimensional rigid rotor}

An even more interesting example of rigid rotor is provided by

$$
\hat{H}=\hat{L}^{2}-g \cos (\theta),
$$

where $\hat{L}^{2}$ is the square of the dimensionless quantum-mechanical angular-momentum operator. This Hamiltonian is invariant under the antiunitary transformation $\hat{A}=\hat{U} \hat{T}$ discussed above when $g$ is purely imaginary.

In order to apply the DM we resort to the set of eigenvectors $|l, m\rangle$ of $\hat{L}^{2}$ and $\hat{L}_{z}$ :

$$
\begin{aligned}
& \hat{L}^{2}|l, m\rangle=l(l+1)|l, m\rangle, \\
& \hat{L}_{z}|l, m\rangle=m|l, m\rangle,
\end{aligned}
$$

where $l=0,1, \ldots$ and $m=0 \pm 1, \pm 2, \ldots, \pm l$ are the angular momentum and magnetic quantum numbers, respectively. Every eigenvector $|\psi\rangle$ of $\hat{H}$ can be expanded as

$$
|\psi\rangle=\sum_{i=0}^{N} c_{i}|M+i, m\rangle,
$$

where $M=|m|$ and the coefficients satisfy the recurrence relation [23] (and references therein)

$$
\begin{aligned}
& A_{i} c_{i-1}+B_{i} c_{i}+A_{i+1} c_{i+1}=0, \\
& A_{i}=-g\left[\frac{i(i+2 M)}{4(i+M)^{2}-1}\right]^{1 / 2}, B_{i}=(i+M)(i+M+1)-E .
\end{aligned}
$$

There is also a simple recurrence relation for the secular determinants [23] but we do not need it here because we can efficiently obtain $E(g)$ from the roots of $c_{N}=0$ for sufficiently large $N$.

We denote $E_{M, n}, M, n=0,1, \ldots$ the eigenvalues of $\hat{H}$ so that $E_{M^{\prime}, n^{\prime}}=E_{M, n}$ when $M+n=M^{\prime}+n^{\prime}$ and $g=0$. The eigenvectors $\left|\psi_{m, n}\right\rangle$ with $m= \pm M$ are degenerate. In the coordinate representation the basis set of eigenvectors of $\hat{L}^{2}$ and $\hat{L}_{z}$ are the spherical harmonics $\langle\theta, \phi \mid l, m\rangle=Y_{l}^{m}(\theta, \phi)$ that satisfy $\hat{A}|l, m\rangle=(-1)^{l}|l,-m\rangle$. 
Besides, it follows from the recurrence relation (27) that $c_{i, M, n}$ is either real or imaginary when $i$ is even or odd, respectively. Therefore, $c_{i, M, n}^{*}(-1)^{M+i}=(-1)^{M} c_{i, M, n}$ and $\hat{A}\left|\psi_{m, n}\right\rangle=(-1)^{M}\left|\psi_{-m, n}\right\rangle$. We clearly see that in this case $\hat{A}\left|\psi_{m, n}\right\rangle \neq \lambda\left|\psi_{m, n}\right\rangle$ but

the eigenvalue $E_{M, n}$ is real because $\hat{H} \hat{A}\left|\psi_{m, n}\right\rangle=E_{M, n} \hat{A}\left|\psi_{m, n}\right\rangle$ in agreement with the more general condition for real eigenvalues developed in section 2 ,

Fig. 3 shows the eigenvalues $E_{M, n}$ for $M=0,1,2,3$ and $n=0,1,2$. It suggests that pairs of eigenvalues $\left(E_{M, 2 n}, E_{M, 2 n+1}\right)$ coalesce at $a= \pm a_{M, n}$ when $g=i a$. Tables 8, 9, 10 and 11 show several critical parameters for $M=0,1,2,3$, respectively . In this case we also find a linear relationship $a_{M, n}=b+c e_{M, n}$ between the critical parameters, where $c \approx 1.18$, and that they increase asymptotically as $n^{2}$.

\section{Conclusions}

It appears to be clear from the results obtained throughout this paper that the DM is a remarkably simple and efficient tool for the calculation of eigenvalues and eigenvectors of a wide class of PT-symmetric models. In fact, the DM appears to converge more rapidly than more elaborate approaches [9] and seems to be particularly useful for the calculation of critical parameters and exceptional points.

The condition for real eigenvalues developed in section 2 appears to be more general than the one invoked in earlier studies of the PT-symmetric Hamiltonians. This fact is plainly illustrated by the perturbed rigid rotator (24) for which the commonly used condition for unbroken symmetry ([6) does not hold but the eigenvalues are real as long as the more general condition (5) applies.

Present numerical investigation suggests that both critical parameters for the three models (10), (20) and (24) behave asymptotically as $n^{2}$. We may be tempted to conjecture that this is a general property of such systems but that is not the case. The analysis of the exactly solvable models with piecewise constant potentials proposed by Znojil and collaborators [11 13 reveals a different behaviour. In the case of the potential $V(x)=i Z x /|x|,-1<x<1$, with Dirichlet or periodic boundary conditions 
at $x= \pm 1$, the critical parameters $e_{n}$ and $Z_{n}$ appear to behave asymptotically as $n^{2}$ and $n$, respectively.

\section{Acknowledgments}

We thank Dr. M. Znojil for useful comments and suggestions that helped to improve this paper

[1] Delabaere E and Pham F 1998 Phys. Lett. A 25029.

[2] Fernández F M, Guardiola R, Ros J, and Znojil M 1998 J. Phys. A 3110105.

[3] Delabaere E and Trinh D A 2000 J. Phys. A 338771.

[4] Bender C M, Berry M, Meisinger P N, Savage V M, and Simsek M 2001 J. Phys. A 34 L31.

[5] Handy C N 2001 J. Phys. A 345065.

[6] Handy C N, Khan D, Wang X-Q, and Tymczak C J 2001 J. Phys. A 345593.

[7] Handy C N and Wang X-Q 2003 J. Phys. A 3611513.

[8] Bender C M and Weir D J 2012 J. Phys. A 45425303.

[9] Handy C N and Vrinceanu D 2013 J. Phys. A 46135202.

[10] Rubinstein J, Sternberg P, and Ma Q 2007 Phys. Rev. Lett. 99167003.

[11] Znojil M 2001 Phys. Lett. A 2857.

[12] Znojil M and Lévai G 2001 Mod. Phys. Lett. A 162273.

[13] Jakubský V and Znojil M 2004 Czech. J. Phys. 541101

[14] Bender C M and Kalveks R J 2011 Int. J. Theor. Phys. 50955.

[15] Heiss W D and Sannino A L 1990 J. Phys. A 231167.

[16] Heiss W D 2000 Phys. Rev. E 61929.

[17] Heiss W D and Harney H L 2001 Eur. Phys. J. D 17149.

[18] Heiss W D 2004 Czech. J. Phys. 541091.

[19] Bender C M, Meisinger P N, and Wang Q 2003 J. Phys. A 361973.

[20] Bender C M, Brod J, Refig A, and Reuter M E 2004 J. Phys. A 3710139.

[21] Wigner E 1960 J. Math. Phys. 1409.

[22] Abramowitz M and Stegun I A 1972 Handbook of Mathematical Functions (Dover, New York).

[23] Fernández F M 2001 Introduction to Perturbation Theory in Quantum Mechanics (CRC Press, Boca Raton). 
Critical parameters for non-hermitian Hamiltonians

Table 1. Convergence of the DM and OPPQ for $\hat{H}=\hat{p}^{2}+i \hat{x}^{3}$

\begin{tabular}{|r|c|c|c|c|}
\hline & DM & OPPQ & DM & OPPQ \\
\hline$N$ & \multicolumn{2}{|c|}{$E_{0}$} & \multicolumn{2}{|c|}{$E_{1}$} \\
\hline 20 & 1.15638348063027 & 1.15720107946295 & 4.10944159217725 & 3.85785039690029 \\
40 & 1.15626708286738 & 1.15626701076546 & 4.10922836311577 & 4.10917078909004 \\
60 & 1.15626707198833 & 1.15626707203003 & 4.10922875272617 & 4.10922884747775 \\
80 & 1.15626707198811 & 1.15626707198786 & 4.10922875280961 & 4.10922875282249 \\
100 & 1.15626707198811 & 1.15626707198811 & 4.10922875280965 & 4.10922875280956 \\
\hline & \multicolumn{2}{|c|}{$E_{2}$} & \multicolumn{2}{|c|}{$E_{3}$} \\
\hline 20 & 7.79277572798155 & 7.27293255888356 & 10.3897589647850 & 10.11399345333521 \\
40 & 7.562284306885572 & 7.56274007348397 & 11.3137218751498 & 11.44673034474738 \\
60 & 7.56227386040027 & 7.56226879749661 & 11.3144217612385 & 11.31447586752061 \\
80 & 7.56227385497881 & 7.56227386127323 & 11.3144218200804 & 11.31442184225783 \\
100 & 7.56227385497882 & 7.56227385497590 & 11.3144218201957 & 11.31442182025857 \\
\hline
\end{tabular}

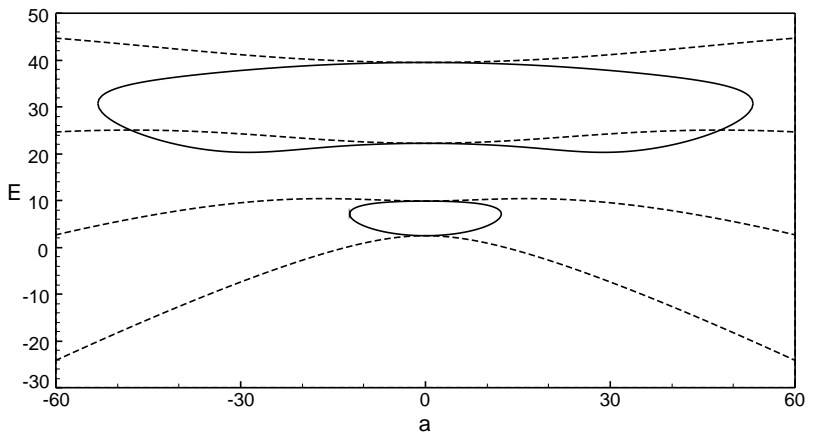

Figure 1. First four eigenvalues $E_{n}(g)$ for the model (10) with $g=a$ (dashed line) and $g=i a$ (solid line) 

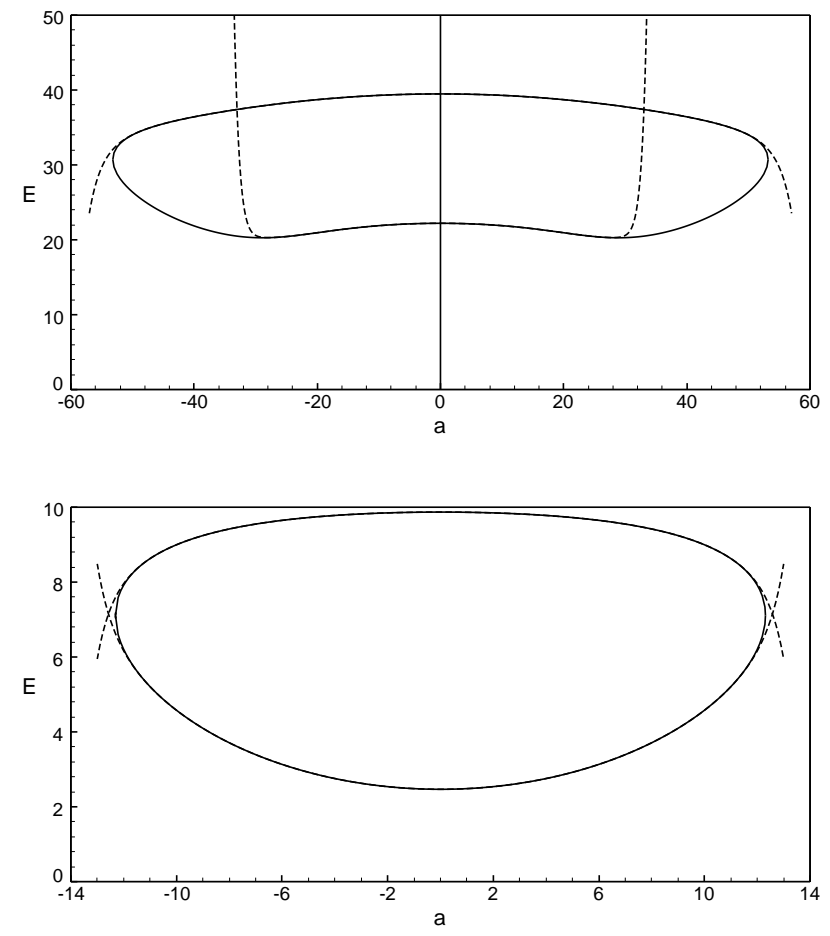

Figure 2. First four eigenvalues $E_{n}(g)$ for the model (10) with $g=i a$ calculated by the diagonalization method (solid line) and perturbation theory (dashed line)
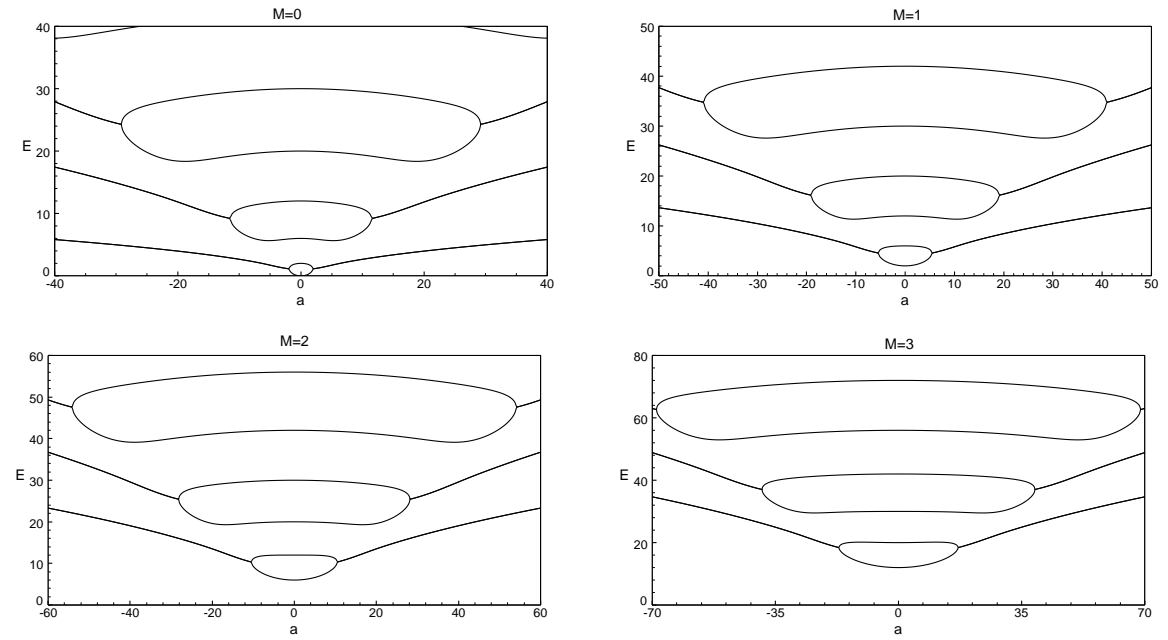

Figure 3. Eigenvalues $E_{M, n}(i a)$ of the rigid rotor (24) 
Critical parameters for non-hermitian Hamiltonians

Table 2. Critical parameters for the oscillator (8)

\begin{tabular}{|c|c|c|}
\hline$n$ & $e_{n}$ & $a_{n}$ \\
\hline 0 & 1.28277353565056613093 & -2.61180935658887732269 \\
1 & 4.18138810077014360384 & -5.37587963413369849339 \\
2 & 7.47676353160394726567 & -7.81513358112177472963 \\
3 & 11.03766256181169489101 & -10.07564704682307238859 \\
4 & 14.80256612165608800708 & -12.21531517192134682450 \\
5 & 18.73495127980953607811 & -14.26484986999511696653 \\
6 & 22.81035758069971715940 & -16.24312145518034341186 \\
7 & 27.01113795189653614640 & -18.16282077195707147474 \\
8 & 31.32389750099022726315 & -20.03302515800852425790 \\
9 & 35.73808590511219720429 & -21.86052509995057604960 \\
10 & 40.24515595690885890435 & -23.65057685885184848462 \\
11 & 44.83802791988671972061 & -25.40736007787328669330 \\
12 & 49.51073146222982552736 & -27.13427141318176849625 \\
13 & 54.25815672832834563937 & -28.83412125570701326472 \\
14 & 59.07587562778958582850 & -30.50927027533271429436 \\
15 & 63.96001004047454636742 & -32.16172704057811933141 \\
16 & 68.9071323790276889544 & -33.7932195834531855763 \\
17 & 73.91418907962735035 & -35.405249007491647104 \\
\hline & 78.9784407229043496 & -36.9991304044614914 \\
\hline
\end{tabular}


Table 3. Critical parameters for the oscillator (9)

\begin{tabular}{|c|c|c|}
\hline$n$ & $e_{n}$ & \multicolumn{1}{|c|}{$a_{n}$} \\
\hline 0 & 3.17338956654721488704 & 3.16903614167472725234 \\
1 & 11.32761640743725703756 & 7.62596008108023132512 \\
2 & 21.47216949764589814716 & 12.11537100311929607154 \\
3 & 33.02428793244591467473 & 16.61105709045349074831 \\
4 & 45.70317143857586670043 & 21.10901685823201530899 \\
5 & 59.33696104179223837682 & 25.60805225319570978500 \\
6 & 73.80750220757362981500 & 30.10768065951909870293 \\
7 & 89.0276216454863021248 & 34.6076704707909122127 \\
8 & 104.9298551095159538 & 39.10789674411541992 \\
9 & 121.460151413651 & 43.6082861575648 \\
10 & 138.5740516383 & 48.10879285437 \\
\hline
\end{tabular}


Critical parameters for non-hermitian Hamiltonians

Table 4. Critical parameters for the box model (10)

\begin{tabular}{|c|l|l|}
\hline$n$ & \multicolumn{1}{|c|}{$e_{n}$} & \multicolumn{1}{|c|}{$a_{n}$} \\
\hline 1 & 7.1085995967646 & 12.3124556722597 \\
2 & 30.70746876678 & 53.18689607587 \\
3 & 70.9578499846 & 122.902601369 \\
4 & 127.862609648 & 221.464536296 \\
5 & 201.42215453 & 348.87340541 \\
6 & 291.6365885 & 505.1293887 \\
7 & 398.5059476 & 690.2325483 \\
8 & 522.030247 & 904.182911 \\
9 & 662.209493 & 1146.98049 \\
10 & 819.043691 & 1418.625286 \\
11 & 992.532842 & 1719.11731 \\
12 & 1182.67695 & 2048.4566 \\
13 & 1389.476009 & 2406.643039 \\
14 & 1612.93003 & 2793.67675 \\
15 & 1853.0390 & 3209.5577 \\
16 & 2109.80293 & 4127.8613 \\
\hline 17 & 2383.22182 & \\
\hline & & \\
\hline
\end{tabular}


Table 5. Critical parameter $a_{1}$ from the perturbation series

\begin{tabular}{|c|c|}
\hline$k$ & $a_{1}(k)$ \\
\hline 9 & 12.31814954 \\
19 & 12.31354496 \\
29 & 12.31290237 \\
39 & 12.31269737 \\
49 & 12.31260686 \\
59 & 12.31255909 \\
69 & 12.31253084 \\
79 & 12.31251277 \\
89 & 12.31250051 \\
99 & 12.31249181 \\
\hline
\end{tabular}


Table 6. Critical parameters for the even states of the Mathieu equation (22)

\begin{tabular}{|c|c|}
\hline$e_{n}$ & $a_{n}$ \\
\hline 2.08869890274969540742210705005 & 1.46876861378514199230729308986 \\
\hline 27.3191276740344351613697285995 & 16.4711658922636564062419622945 \\
\hline 80.6582642367217733231182880374 & 47.8059657025975746007950854808 \\
\hline 162.107021116501331382763597087 & 95.4752727072182593469528060868 \\
\hline 271.665574614890515399359662310 & 159.479212669357057187230627715 \\
\hline 409.333979844643194402422763806 & 239.817810495650789094138995905 \\
\hline 575.112259376089614140747231520 & 336.491073930202402676797136666 \\
\hline 769.000424132277697815886582932 & 449.499006061556590915787589874 \\
\hline 990.998480035440536142042914292 & 578.841608335703329386650346074 \\
\hline 1241.10643057248550485513720070 & 724.518881510280902738995966517 \\
\hline 1519.32427792923873387283500008 & 886.530826016874701071963928710 \\
\hline 1825.65202354516187327873747825 & 1064.87744211774801292171298635 \\
\hline 2160.08966840670531841142295477 & 1259.55872998069003603099135522 \\
\hline 2522.63721321244381656497120868 & 1470.57468971764989052881373681 \\
\hline 2913.29465847091131070672526973 & 1697.92532140597167537370468979 \\
\hline 3332.06200456108886585906171039 & 1941.61062510068817839368674171 \\
\hline 3778.93925177117865219161016434 & 2201.63060084195557145589058358 \\
\hline 4253.92640032424228639948323784 & 2477.98524865972002792020985438 \\
\hline 4757.02345039561656754162219537 & 2770.67456857674245292672357391 \\
\hline 5288.23040212502649969662284873 & 3079.69856061061467026098084225 \\
\hline
\end{tabular}


Table 7. Critical parameters for the odd states of the Mathieu equation (22)

\begin{tabular}{|c|c|}
\hline$e_{n}$ & $a_{n}$ \\
\hline 11.1904735991293865896020980123 & 6.92895475876018147964342787950 \\
\hline 50.4750161557597516452005364504 & 30.0967728375875542000339071418 \\
\hline 117.868924160843684783814608183 & 69.5987932768953947914148570394 \\
\hline 213.372568637479279993815862834 & 125.435411314308272709560436718 \\
\hline 336.986043950205287207567051913 & 197.606678692480922034682411560 \\
\hline 488.709384475887730016940247407 & 286.112608761678078262070275163 \\
\hline 668.542605654162967762763559437 & 390.953206295596779988894940683 \\
\hline 876.485715432799125784653813063 & 512.128473373035028002129394632 \\
\hline 1112.53871831587949363459807537 & 649.638411028231983524090563574 \\
\hline 1376.70161704521717624727857705 & 803.483019827838526685397002241 \\
\hline 1668.97441338489968248739617901 & 973.662300105893000632623663802 \\
\hline 1989.35710852120412013890118346 & 1160.17625207096144146935385017 \\
\hline 2337.84970328115567018520806164 & 1363.02487585944750618204515605 \\
\hline 2714.45219825889803861462924172 & 1582.20817156403242791167234121 \\
\hline 3119.16459389224590002123285708 & 1817.72613924973691186689894477 \\
\hline 3551.98689051091222035608251051 & 2069.57877896343363081378199317 \\
\hline 4012.91908836792421411986633224 & 2337.76609073971215299835857492 \\
\hline 4501.96118766068778990529756079 & 2622.28807460462195641810433326 \\
\hline 5019.11318854547174684532365214 & 2923.14473057813385462524614741 \\
\hline 5564.37509114759075160843417379 & 3240.33605867580166867491717218 \\
\hline 6137.74689556870672683751212500 & 3573.86205890991029411196172754 \\
\hline 6739.22860189215699095301722791 & 3923.72273129028543611909139321 \\
\hline 7368.82021018690440596500439191 & 4289.91807582487536028648347609 \\
\hline
\end{tabular}


Table 8. Critical parameters $e_{0, n}$ and $a_{0, n}$ for the rigid rotor (24)

\begin{tabular}{|c|c|}
\hline$e_{0, n}$ & $a_{0, n}$ \\
\hline 1.11850860747789604879129584124 & 1.89945169187324547365901350058 \\
\hline 9.18271110777602614314692313478 & 11.4469373135041414112902268409 \\
\hline 24.2743374650550706797661994079 & 29.1570364187843312750194317104 \\
\hline 46.3934021737006494552531157256 & 55.0338230301496191682912997241 \\
\hline 75.5399201827232487162615410925 & 89.0777654885162317220462901731 \\
\hline 111.713900380652159264985603687 & 131.288974351497399022703273189 \\
\hline 154.915347674365981794080626693 & 181.667486575132082638246802870 \\
\hline 205.144264889286269484450927321 & 240.213317402561182509835277416 \\
\hline 262.400653742394747193838743959 & 306.926474080239226003553463095 \\
\hline 326.684515329643806117627299256 & 381.806960425731499957841447183 \\
\hline 397.995850380696676312538960541 & 464.854778613145237343458945801 \\
\hline 476.334659399023202928517978051 & 556.069929958740540140414381845 \\
\hline 561.700942742735329186201508058 & 655.452415299711583081531438128 \\
\hline 654.094700673266377544747681781 & 763.002235190629698442777914673 \\
\hline 753.515933385805509695023538647 & 878.719390011577165280746746226 \\
\hline 859.964641028959999602185575728 & 1002.60388003069997387686020878 \\
\hline 973.440823717829191452457184709 & 1134.65570544194626000993141955 \\
\hline 1093.94448154292133694845436910 & 1274.87486638866994863623150054 \\
\hline 1221.47561457637370003885489988 & 1423.26136297886278858803945222 \\
\hline 1356.03422287637955815251177760 & 1579.81519529525700149353590512 \\
\hline 1497.62030649039627961298586330 & 1744.53636340218999765413768412 \\
\hline 1646.23386545750814120158073731 & 1917.42486735037046169355652365 \\
\hline 1801.87489981019236211810598331 & 2098.48070718025182357309767219 \\
\hline 1964.54340957565684861430612511 & 2287.70388292446187324632564614 \\
\hline 2134.23939477686596016391844190 & 2485.09439460958034550662389867 \\
\hline 2310.96285543333590341463022204 & 2690.65224225745820774568590806 \\
\hline 2494.71379156175787158357548067 & 2904.37742588620969831042658859 \\
\hline
\end{tabular}


Table 9. Critical parameters $e_{1, n}$ and $a_{1, n}$ for the rigid rotor (24)

\begin{tabular}{|c|c|}
\hline$e_{1, n}$ & $a_{1, n}$ \\
\hline 4.55877886725924641484810680290 & 5.41369967947421154076411805664 \\
\hline 16.1375907539446796948176665321 & 19.0366539366410365977084445332 \\
\hline 34.7430624620380644472582121023 & 40.8287653735067375076502059995 \\
\hline 60.3758571013293211953590679065 & 70.7886035789264845417420851348 \\
\hline 93.0360844044641996314893055308 & 108.915912141697487819521883860 \\
\hline 132.723772524583231646115964443 & 155.210615993212798290721507998 \\
\hline 179.438930647130853022234048182 & 209.672686433039528417476556225 \\
\hline 233.181562239157762078784983517 & 272.302110446390569333909532764 \\
\hline 293.951668731053476959597684378 & 343.098881384146395386662172917 \\
\hline 361.749250740754368699280246494 & 422.062995536098442607803489884 \\
\hline 436.574308535710155676251436297 & 509.194450686347541286810153760 \\
\hline 518.426842224132039104965417532 & 604.493245438036181898256235869 \\
\hline 607.306851839808768157712103699 & 707.959378871064297125726282783 \\
\hline 703.214337381672878552754182737 & 819.592850356874342990995371324 \\
\hline 806.149298832917264169042646291 & 939.393659452681323113486417084 \\
\hline 916.111736170419252202468288054 & 1067.36180583827770608337708586 \\
\hline 1033.10164936940069761597136643 & 1203.49728927679401104146044559 \\
\hline 1157.11903840568460024469637942 & 1347.80010958951204915746935249 \\
\hline 1288.16390325671987308020948612 & 1500.27026663922414661704954168 \\
\hline 1426.23624390197230137796623183 & 1660.90776031895581341708604925 \\
\hline 1571.33606032299333370614104097 & 1829.71259054414926566147073681 \\
\hline 1723.46335250333125098536155945 & 2006.68475724713587692441388188 \\
\hline 1882.61812042837198303912549092 & 2191.82426037315622876377594946 \\
\hline 2048.80036408515554263447292272 & 2385.13109987744749454382949289 \\
\hline 2222.01008346219172139310274467 & 2586.60527572308025767686172501 \\
\hline 2402.24727854928656430083016851 & 2796.24678787933020022750968876 \\
\hline 2589.51194933738457410429640333 & 3014.05563632043725171822918588 \\
\hline
\end{tabular}


Table 10. Critical parameters $e_{2, n}$ and $a_{2, n}$ for the rigid rotor (24)

\begin{tabular}{|c|c|}
\hline$e_{2, n}$ & $a_{2, n}$ \\
\hline 10.3208166747903646568973037932 & 10.4288550159906556880861532857 \\
\hline 25.4207623327887544466386812765 & 28.1582740390332761901432188657 \\
\hline 47.5418883505162195939968735032 & 54.0402932178718093860340915287 \\
\hline 76.6891413244321657490533380555 & 88.0863457427007731538106071227 \\
\hline 112.863428447014039279577496571 & 130.298593278713537202154514528 \\
\hline 156.065011915265846305454988719 & 180.677682922350525891381170392 \\
\hline 206.293988039332160271025550914 & 239.223862208215672094273506007 \\
\hline 263.550397974137409898781499507 & 305.937241931464725274206268463 \\
\hline 327.834261238895647696546524794 & 380.817877474579788921392992095 \\
\hline 399.145587817865851310066047948 & 463.865798896565719257861484418 \\
\hline 477.484383113449595517060626653 & 555.081023588338983061868169447 \\
\hline 562.850650174688796531422680455 & 654.463562138981667213774784976 \\
\hline 655.244390776764559156247145443 & 762.013421271343462077625746903 \\
\hline 754.665605975802226624019198276 & 877.730605405071181726830864340 \\
\hline 861.114296408136089048224877344 & 1001.61511753266849630211224248 \\
\hline 974.590462458325714246783348069 & 1133.66695973221802998481790522 \\
\hline 1095.09410435669169203890272295 & 1273.88613347875288396645703396 \\
\hline 1222.62522223754679315385272073 & 1422.27263983948977169496533497 \\
\hline 1357.18381617478886848820532514 & 1578.82647959970741927897388563 \\
\hline 1498.76988620408725276163261494 & 1743.54765334592907523155490638 \\
\hline 1647.38343233693679094718818617 & 1916.43616152210473017073444202 \\
\hline 1803.02445456967522723386079799 & 2097.49200446830527099050583774 \\
\hline 1965.69295288932481713767155384 & 2286.71518244784416497102175928 \\
\hline 2135.38892727740046871358920607 & 2484.10569566659182256233911612 \\
\hline 2312.11237771239876092876125824 & 2689.66354428692976622447303147 \\
\hline 2495.86330417142194483897972208 & 2903.38872843796560070724063761 \\
\hline 2686.64170663122979954448053178 & 3125.28124822310130537164659655 \\
\hline
\end{tabular}


Table 11. Critical parameters $e_{3, n}$ and $a_{3, n}$ for the rigid rotor (24)

\begin{tabular}{|c|c|}
\hline$e_{3, n}$ & $a_{3, n}$ \\
\hline 18.3932656869754919346793973788 & 16.8966533642743226378461806280 \\
\hline 37.0261276648638864684719643139 & 38.7837061748124744181847563089 \\
\hline 62.6669859858232821773304748336 & 68.7740586891067799587798103556 \\
\hline 95.3306215019736927494865643118 & 106.915119263007675368240263857 \\
\hline 135.020028981874691707132974862 & 153.217194854387105286541377321 \\
\hline 181.736151824585196120205263944 & 207.683669335464793838124652103 \\
\hline 235.479364255103716530989139084 & 270.315929035220727532388027826 \\
\hline 296.249838077915056335119725000 & 341.114629238351091770190392543 \\
\hline 364.047660655340315855748376441 & 420.080112725581765701401257790 \\
\hline 438.872879915491109161811406148 & 507.212572987544823448482273980 \\
\hline 520.725523743932161740530113278 & 602.512125938872178124628573208 \\
\hline 609.605609143244375059516152739 & 705.978844426605700033807188021 \\
\hline 705.513146883842805626496171499 & 817.612776073132832143973505231 \\
\hline 808.448144007708646910693123011 & 937.413953057538062250373844424 \\
\hline 918.410605243078958607010397392 & 1065.38239774458967328397480722 \\
\hline 1035.40053383702430388114116904 & 1201.51812605992292426362249599 \\
\hline 1159.41793206278032717306053303 & 1345.82114958635061001956413623 \\
\hline 1290.46280153831843226127329649 & 1498.29147690625339213241310920 \\
\hline 1428.53514343169852409564937760 & 1658.92911448450732063468893814 \\
\hline 1573.63495859652721769067726115 & 1827.73406726311001705562366049 \\
\hline 1725.76224766315409747995008471 & 2004.70633907016901625781758106 \\
\hline 1884.91701110119625582112832348 & 2189.84593290656915762534485743 \\
\hline 2051.09924926310976011661273016 & 2383.15285115035342904111463156 \\
\hline 2224.30896241500183043304906063 & 2584.62709570470366379109882661 \\
\hline 2404.54615075871058072339672775 & 2794.26866810660184529365303827 \\
\hline 2591.81081444781812982947448859 & 3012.07756960765198814361352503 \\
\hline 2786.10295359939116923987354036 & 3238.05380123490915423799786847 \\
\hline
\end{tabular}

